\title{
Dog vaccination with EgM proteins against Echinococcus granulosus
}

\author{
Zhuang-Zhi Zhang ${ }^{1 \dagger}$, Gang Guo ${ }^{2 \dagger}$, Jun Li², Bao-Xin Shi', Li Zhao', Bao-Ping Guo ${ }^{2}$, Xu Zhang ${ }^{1}$, Jun-Wei Wang ${ }^{1}$,
} Xue-Ting Zheng' ${ }^{2}$, Wen-Jing $\mathrm{Qi}^{2}$, Li He ${ }^{2}$ and Wen-Bao Zhang ${ }^{2 *}$

\begin{abstract}
Background: Dogs play a pivotal role in the transmission of cystic echinococcosis (CE), a zoonosis caused by the tapeworm Echinococcus granulosus. We showed previously that dogs vaccinated with two E. granulosus adult-worm specific proteins, EgM9 and EgM123, emulsified with Freund's adjuvants induced significant protective efficacy in terms of reduction in worm burden and egg production after 45 days post-infection. It was not known whether this protection can be sustained using adjuvants suitable for use in dogs.

Methods: Recombinant EgM9 and EgM123 were mixed with Quil A or ISCOMs for vaccinating dogs. After three vaccine injections, all the dogs were orally challenge-infected with 200000 protoscoleces of $E$. granulosus. After 45 days of infection, all the dogs were euthanized and necropsied for collecting and counting E. granulosus worms. Immunoglobins, including the $\lg G$ subclasses $\lg G 1$ and $\lg G 2$, were detected in the sera of vaccinated dogs by ELISA. To determine whether the protection efficacy could be maintained after 45 days post-infection, we implemented a longevity trial to count eggs in dog faeces for 170 days after infection.

Results: The dogs vaccinated with EgM9 and EgM123 mixed with Quil A and ISCOMs showed similar protective efficacy as the proteins emulsified with Freund's adjuvants in our previous study in terms of reduction of worms and eggs at 45 days post-infection. The longevity trial showed that EgM9 protein-vaccinated group released lower number of eggs per gram compared with the egg counts in the control dogs during the dog trial study.
\end{abstract}

Conclusion: EgM9 and EgM123 are thus suitable vaccine candidates against E. granulosus infection in dogs.

Keywords: Echinococcus granulosus, EgM proteins, Dog vaccine, Adjuvant

\section{Multilingual abstract}

Please see Additional file 1 for translations of the abstract into the five official working languages of the United Nations.

\section{Background}

Cystic echinococcosis (CE) is caused by the larval stage of the dog tapeworm Echinococcus granulosus and is increasingly recognized as a major public health problem globally $[1,2]$. Given the wide endemicity and severity of the disease, the World Health Organisation (WHO) has included $\mathrm{CE}$ as one of the 17 neglected diseases in its

\footnotetext{
*Correspondence: wenbaozhang2013@163.com

'Zhuang-Zhi Zhang and Gang Guo contributed equally to this work.

${ }^{2}$ State Key Laboratory of Pathogenesis, Prevention and Treatment of High Incidence Diseases in Central Asia, Clinical Medicine Institute, The first Affiliated Hospital of Xinjiang Medical University, Urumqi 830054, Xinjiang, China Full list of author information is available at the end of the article
}

strategic roadmap for 2020 [3]. However, CE is difficult to control in most continental endemic areas. Dogs are major definitive hosts for E. granulosus and play a pivotal role in the transmission of echinococcosis to humans. Vaccination of dogs against E. granulosus infection provides an acceptable and cost-effective complementary means to control echinococcosis as there are far fewer dogs than sheep on farms [4,5]. In our previous studies, we isolated three genes, termed EgM4, EgM9 and EgM123 from mature adult worm of E. granulosus [6], and two proteins, EgM9 and EgM123 emulsified with Freund's adjuvant induced protective efficacy against $E$. granulosus infection in dogs in terms of reduction in worm burden and egg production after 45 days postinfection [7]. However, Freund's adjuvant is not a suitable adjuvant for vaccination of dogs. In the present study, we used Quil A as adjuvant to confirm the vaccine efficacy of

(C) The Author(s). 2018 Open Access This article is distributed under the terms of the Creative Commons Attribution 4.0 International License (http://creativecommons.org/licenses/by/4.0/), which permits unrestricted use, distribution, and 
the EgM proteins. We also used commercial ISCOMs as an adjuvant for the EgM protensagainst infection. In addition, we checked E. granulosus eggs in the faeces of dogs for 170 days post-infection to test the longevity of protection induced by EgM9 and EgM123.

\section{Methods}

\section{Dogs for vaccination trials}

Dogs were purchased from two resources: 1) Beagles (aged 8-12 months) were purchased from Shanghai and Guangdong Animal Centres; and 2) dogs (local strain, 820 months) were from areas in Xinjiang where a hydatid disease control program had been in operation mainly through dosing dogs monthly with praziquantel for a number of years. Dogs without tapeworm infection were selected having been tested used the purging method [8]. In each of the trials, dogs, half male and half female, were randomly divided into each group. The number of dogs in each group are shown in Tables 1,2 and 3. All the dogs were housed in a quarantine facility at the Veterinary Research Institute, Xinjiang Academy of Animal Sciences, Urumqi, China and were maintained on dog biscuits, and tap water.

\section{Protein expression and purification}

The expression and purification of EgM4, EgM9 and EgM123 fused with GST (EgM4-GST, EgM9-GST and EgM123-GST) have been described in our previous studies [6,7]. To test antibody levels, we inserted the EgM genes into the pET28 vector (Novagen). The recombinant vectors were then transformed into $E$. coli (BL21 strain) to express EgM4, EgM9 and EgM123 fused with a 6-His tag. The proteins were purified using affinity columns (Novagen) and then coated onto ELISA plates with $100 \mu \mathrm{l} /$ well at a concentration of $0.5 \mu \mathrm{g} / \mathrm{ml}$ to measure antibody levels. The details of all methods employed, including serum preparation and the ELISA protocol have been described [7].

Table 1 E. granulosus worm burdens at day 45 post-infection in Beagles vaccinated with rEgM9/ Quil A adjuvant

\begin{tabular}{|c|c|c|c|c|c|c|c|c|}
\hline \multirow[t]{2}{*}{ Group (protein) } & \multirow[t]{2}{*}{ Dog No. } & \multicolumn{7}{|c|}{ Number of worms } \\
\hline & & Total & $\leq 3 \mathrm{seg}$ & $\%$ & $\geq 4 \mathrm{seg}$ & $\%$ & Gravid Seg & $\%$ \\
\hline \multirow[t]{11}{*}{ GST controls } & 71122 & 17154 & 8025 & 46.8 & 7445 & 43.4 & 1684 & 9.8 \\
\hline & 71123 & 43725 & 16425 & 37.6 & 17833 & 40.8 & 9467 & 21.7 \\
\hline & 71124 & 14543 & 4908 & 33.7 & 7452 & 51.2 & 2183 & 15.0 \\
\hline & 71125 & 34779 & 14792 & 42.5 & 15162 & 43.6 & 4825 & 13.9 \\
\hline & 71126 & 18909 & 9233 & 48.8 & 8267 & 43.7 & 1409 & 7.5 \\
\hline & 71127 & 32606 & 16333 & 50.1 & 8867 & 27.2 & 7406 & 22.7 \\
\hline & 71129 & 19204 & 6358 & 33.1 & 9912 & 51.6 & 2934 & 15.3 \\
\hline & Average & 25846 & 10868 & 42.0 & 10705 & 41.4 & 4273 & 16.5 \\
\hline & $S D$ & 11111 & 4878 & - & 4120 & - & 3114 & - \\
\hline & Median & 19204 & 9233 & 48.1 & 8867 & 46.2 & 2934 & 15.3 \\
\hline & $\mathrm{IQR}$ & $(17154,34779)$ & $(6358,16333)$ & - & $(7452,15162)$ & - & $(1684,7406)$ & - \\
\hline \multirow[t]{13}{*}{ EgM9 } & 71112 & 21008 & 20496 & 97.6 & 512 & 2.4 & 0 & 0.0 \\
\hline & 71113 & 9229 & 8475 & 91.8 & 754 & 8.2 & 0 & 0.0 \\
\hline & 71114 & 9340 & 4588 & 49.1 & 4752 & 50.9 & 0 & 0.0 \\
\hline & 71115 & 12824 & 12342 & 96.2 & 482 & 3.8 & 0 & 0.0 \\
\hline & 71116 & 1558 & 1558 & 100.0 & 0 & 0.0 & 0 & 0.0 \\
\hline & 71117 & 28801 & 19651 & 68.2 & 8783 & 30.5 & 367 & 1.3 \\
\hline & 71128 & 30746 & 26083 & 84.8 & 4167 & 13.6 & 496 & 1.6 \\
\hline & Average & 16215 & 13313 & 82.1 & 2779 & 17.1 & 123 & 0.8 \\
\hline & $S D$ & 10916 & 9069 & - & 3269 & - & 214 & - \\
\hline & Median & 12824 & 12342 & 96.2 & 754 & 5.9 & 0 & 0 \\
\hline & $\mathrm{IQR}$ & $(9229,28801)$ & $(4588,20496)$ & - & $(482,4752)$ & - & $(0,367)$ & - \\
\hline & Redu \% ${ }^{\mathrm{a}}$ & 33.2 & - & - & - & - & 100 & - \\
\hline & $P$ value $^{b}$ & 0.128 & 0.655 & - & 0.004 & - & 0.001 & - \\
\hline
\end{tabular}

${ }^{a}$ Compared to those in the GST group. Redu, reduction = (worm burden median in GST-worm burden median in the experiment group)/worm burden median in GST group $\times 100 \%$. ${ }^{\text {b }}$ The Mann-Whitney $U$ test was used to compare the worm burden median. $P$ value less than 0.05 means that reduction is significant between GST and experimental groups using median analysis. The meaning of ${ }^{a}$, $b_{\text {is }}$ the same in the following tables 
Table 2 E. granulosus worm burdens at day 45 post-infection with in dogs (local Xinjiang breed) vaccinated with rEgM4 and rEgM123 proteins mixed with Quil A

\begin{tabular}{|c|c|c|c|c|c|c|c|c|}
\hline \multirow[t]{2}{*}{ Group (protein) } & \multirow[t]{2}{*}{ Dog No. } & \multicolumn{7}{|c|}{ Number of worms } \\
\hline & & Total & $\leq 3 \mathrm{seg}$ & $\%$ & $\geq 4 \mathrm{seg}$ & $\%$ & Gravid Seg & $\%$ \\
\hline \multirow[t]{16}{*}{ GST } & 815 & 10228 & 1063 & 10.4 & 9165 & 89.6 & 0 & 0.0 \\
\hline & 817 & 17897 & 8103 & 45.3 & 7528 & 42.1 & 2266 & 12.7 \\
\hline & 821 & 22505 & 677 & 3.0 & 21828 & 97.0 & 0 & 0.0 \\
\hline & 822 & 27892 & 11151 & 40.0 & 9529 & 34.2 & 7212 & 25.9 \\
\hline & 829 & 42724 & 18311 & 42.9 & 12532 & 29.3 & 11881 & 27.8 \\
\hline & 831 & 26808 & 18762 & 70.0 & 8046 & 30.0 & 0 & 0.0 \\
\hline & 833 & 9484 & 839 & 8.8 & 8645 & 91.2 & 0 & 0.0 \\
\hline & 834 & 34147 & 15618 & 45.7 & 12085 & 35.4 & 6444 & 18.9 \\
\hline & 837 & 11053 & 927 & 8.4 & 10126 & 91.6 & 0 & 0.0 \\
\hline & 841 & 47079 & 19658 & 41.8 & 17424 & 37.0 & 9997 & 21.2 \\
\hline & 842 & 31342 & 20582 & 65.7 & 4099 & 13.1 & 6661 & 21.3 \\
\hline & 843 & 13535 & 6178 & 45.6 & 4237 & 31.3 & 3120 & 23.1 \\
\hline & Average & 24558 & 10156 & 41.4 & 10437 & 42.5 & 3965 & 16.1 \\
\hline & $S D$ & 12708 & 8174 & - & 5083 & - & 4319 & - \\
\hline & Median & 24657 & 9627 & 39.0 & 9347 & 37.9 & 2693 & 10.9 \\
\hline & IQR & $(11674,33446)$ & $(961,18649)$ & & $(7787,2309)$ & & $(0,7074)$ & - \\
\hline \multirow[t]{15}{*}{ EgM4 } & 813 & 34908 & 1805 & 5.2 & 25050 & 71.8 & 8053 & 23.1 \\
\hline & 816 & 0 & 0 & 0 & 0 & 0.0 & 0 & 0.0 \\
\hline & 818 & 11624 & 3256 & 28.0 & 6271 & 53.9 & 2097 & 18.0 \\
\hline & 819 & 0 & 0 & 0 & 0 & 0.0 & 0 & 0.0 \\
\hline & 820 & 14938 & 12469 & 83.5 & 1696 & 11.4 & 773 & 5.2 \\
\hline & 826 & 9939 & 7982 & 80.3 & 1103 & 11.1 & 854 & 8.6 \\
\hline & 832 & 0 & 0 & 0 & 0 & 0.0 & 0 & 0.0 \\
\hline & 838 & 1796 & 1796 & 100.0 & 0 & 0.0 & 0 & 0.0 \\
\hline & 840 & 39773 & 27124 & 68.2 & 8897 & 22.4 & 3752 & 9.4 \\
\hline & Average & 18830 & 9072 & 48.2 & 7170 & 38.1 & 2588 & 13.7 \\
\hline & $S D$ & 15056 & 9779 & - & 9399 & - & 2982 & - \\
\hline & Median & 9939 & 1805 & 18.2 & 1103 & 11.1 & 773 & 7.8 \\
\hline & IQR & $(0,24923)$ & $(0,10225)$ & - & $(0,7584)$ & - & $(0,2925)$ & - \\
\hline & Redu $\%^{\mathrm{a}}$ & 59.7 & 81.3 & - & 88.2 & - & 71.3 & - \\
\hline & $P$ value $^{b}$ & 0.069 & 0.219 & - & 0.013 & - & 0.442 & - \\
\hline \multirow[t]{11}{*}{ EgM123 } & 814 & 128 & 128 & 100.0 & 0 & 0.0 & 0 & 0.0 \\
\hline & 823 & 1075 & 1075 & 100.0 & 0 & 0.0 & 0 & 0.0 \\
\hline & 824 & 0 & 0 & 0 & 0 & 0.0 & 0 & 0.0 \\
\hline & 825 & 0 & 0 & 0 & 0 & 0.0 & 0 & 0.0 \\
\hline & 827 & 3198 & 3198 & 100.0 & 0 & 0.0 & 0 & 0.0 \\
\hline & 828 & 3494 & 3494 & 100.0 & 0 & 0.0 & 0 & 0.0 \\
\hline & 835 & 0 & 0 & 0 & 0 & 0.0 & 0 & 0.0 \\
\hline & 836 & 408 & 408 & 100.0 & 0 & 0.0 & 0 & 0.0 \\
\hline & 839 & 0 & 0 & 0 & 0 & 0.0 & 0 & 0.0 \\
\hline & Average & 1661 & 1661 & 100.0 & 0 & 0.0 & 0 & 0.0 \\
\hline & $S D$ & 1580 & 1580 & - & 0 & - & 0 & - \\
\hline
\end{tabular}


Table 2 E. granulosus worm burdens at day 45 post-infection with in dogs (local Xinjiang breed) vaccinated with rEgM4 and rEgM123 proteins mixed with Quil A (Continued)

\begin{tabular}{|c|c|c|c|c|c|c|c|c|}
\hline \multirow[t]{2}{*}{ Group (protein) } & \multirow[t]{2}{*}{ Dog No. } & \multicolumn{7}{|c|}{ Number of worms } \\
\hline & & $\overline{\text { Total }}$ & $\leq 3 \mathrm{seg}$ & $\%$ & $\geq 4$ seg & $\%$ & Gravid Seg & $\%$ \\
\hline & Median & 128 & 128 & - & 0 & - & 0 & - \\
\hline & IQR & $(0,2137)$ & $(0,2137)$ & - & $(0,0)$ & - & $(0,0)$ & - \\
\hline & Redu\% ${ }^{\mathrm{a}}$ & 99.5 & 98.7 & 0 & 100 & - & 100 & - \\
\hline & $P$ value $^{\mathrm{b}}$ & 0.000 & 0.002 & - & 0.000 & - & 0.023 & - \\
\hline
\end{tabular}

${ }^{a}$ Compared to those in the GST group. Redu, reduction = (worm burden median in GST-worm burden median in the experiment group)/worm burden median in GST group $\times 100 \%$. ${ }^{\text {b }}$ The Mann-Whitney $U$ test was used to compare the worm burden median. $P$ value less than 0.05 means that reduction is significant between GST and experimental groups using median analysis. The meaning of ${ }^{a}$, ${ }$ is the same in the following tables

\section{Vaccination and parasite challenge}

Two trials were carried out for testing the protective efficacy of the EgM proteins. In trial I, two groups each with seven Beagles were used. One group was vaccinated with EgM9-GST as an experimental group and the other with GST mixed with Quil A as a control group. One dose of vaccine comprised $100 \mu \mathrm{g}$ of soluble recombinant EgM9GST or GST and $100 \mu \mathrm{g}$ of Quil A (Superfos Biosector, Demark) in $250 \mu \mathrm{l}$ of PBS. The mixture was stirred overnight at $4{ }^{\circ} \mathrm{C}$ before vaccination. Trial II used Xinjiang local dogs and involved three groups including Group 1 consisting of 12 dogs vaccinated with GST; Group II consisted of nine dogs vaccinated with EgM4 and Group III had nine dogs vaccinated with EgM123. All proteins were mixed with Quil A. The dogs received one primary vaccination and two booster vaccinations by subcutaneous injections with intervals of 2 weeks between each vaccination.

In addition, we mixed $100 \mu \mathrm{g}$ of EgM9-GST, EgM123GST or GST recombinant proteins with $100 \mu \mathrm{g}$ of ISCOMs (AbISCO- 100, Isconova AB, Sweden) and used the preparation to vaccinate dogs of the Xinjiang local breed.

E. granulosus protoscoleces were collected from sheep livers in a slaughterhouse in Urumqi, in western China as previously described [7]. All dogs were orally infected with 200000 protoscoleces of E. granulosus 1 week after the second booster injection.

All the dogs in the above three trials were euthanized and necropsied 45-46 days after infection for collecting and counting E. granulosus worms as described in detail previously [7].

\section{Longevity trial and fecal egg counts}

For the longevity trial, two experimental groups of Beagles were vaccinated with EgM9 $(n=9)$ and EgM123 $(n=10)$ mixed with Quil, using the same dose and schedule as above. Two weeks after the last vaccination boost, all dogs were orally challenged with 200000 protoscoleces. Control dogs $(n=10)$ were vaccinated with only Quil A in PBS. At 40 days post-challenge infection, dog fecal samples were collected every 2 days for the first month and then, every 4 days until 169 days postinfection. To count the number of E. granulosus eggs,
3-5 g of fresh dog faeces was weighed and placed into a Falcon tube. Three glass balls $(6 \mathrm{~mm}$ in diameter) and $25 \mathrm{ml}$ saline were added to the tube. After shaking the tube for $1 \mathrm{~min}$, the faecal sample was passed through a 100 mesh sieve and rinsed with $50 \mathrm{ml}$ saline. The runthrough was centrifuged at $1500 \mathrm{~g}$ for $10 \mathrm{~min}$. After discarding the supernatant, the pellet was washed three times with $50 \mathrm{ml}$ saline and centrifuged at $1500 \mathrm{~g}$ for $10 \mathrm{~min}$. The pellet was then re-suspended in $45 \mathrm{ml}$ of saturated sucrose (1300 g of sucrose in $1000 \mathrm{ml}$ of water) to float the E. granulosus eggs. After $90 \mathrm{~min}$ at room temperature, the top $30 \mathrm{ml}$ of the supernatant was transferred into a fresh Falcon tube containing $20 \mathrm{ml}$ of water. The tube was then centrifuged at $3000 \mathrm{~g}$ for $10 \mathrm{~min}$ to sediment the eggs. After washing twice with $45 \mathrm{ml}$ saline and centrifugation at $1500 \mathrm{~g}$ for $10 \mathrm{~min}$, the pellet was resuspended in $10 \mathrm{ml}$ of saline. Egg numbers were counted using a McMaster slide by adding $0.15 \mathrm{ml}$ per chamber. Ten chambers were counted for each of the dog faecal samples containing high number of eggs and the whole preparation was used to count eggs in each stool sample with low or no eggs. Eggs per gram (EPG) of dog faeces were used in statistical analysis. All dogs were euthanized and necropsied at day 169 or 170 post-infection.

\section{Data collection and analysis}

The Mann-Whitney $U$ test was used to compare worm burdens and eggs per gram (EPG) in experimental and control groups using SPSS software (release 10.0; SPSS, USA). We used median value in each of the groups (inter-quartile range, IQR) for calculation of reduction in worm numbers and eggs. Spearman's rank correlation was used to analyse the correlation between worm burden and serum optical-density values in ELISA. $P<0.05$ was taken to indicate a statistically significant difference.

\section{Results and discussion}

\section{Vaccine efficacy of EgM proteins combined with Quil A}

In trial I, we vaccinated dogs with EgM9 fused with GST mixed with Quil A and we used recombinant GST mixed with Quil A as a control. No significant differences were 
Table 3 E. granulosus worm burdens at day 45 post-infection with in dogs (local Xinjiang breed) vaccinated with rEgM9 and rEgM123 proteins coupled with ISCOMs

\begin{tabular}{|c|c|c|c|c|c|c|c|c|}
\hline \multirow[t]{2}{*}{ Group (protein) } & \multirow[t]{2}{*}{ Dog No. } & \multicolumn{7}{|c|}{ Number of worms } \\
\hline & & Total & $\leq 3 \mathrm{seg}$ & $\%$ & $\geq 4 \mathrm{seg}$ & $\%$ & Gravid Seg & $\%$ \\
\hline \multirow[t]{9}{*}{ GST control } & 802 & 24618 & 24618 & 100.0 & 0 & 0.0 & 0 & 0.0 \\
\hline & 810 & 15273 & 15273 & 100.0 & 0 & 0.0 & 0 & 0.0 \\
\hline & 811 & 2784 & 2784 & 100.0 & 0 & 0.0 & 0 & 0.0 \\
\hline & 1313 & 41288 & 22857 & 55.4 & 18431 & 44.6 & 0 & 0.0 \\
\hline & 1314 & 11547 & 7362 & 63.8 & 4185 & 36.2 & 0 & 0.0 \\
\hline & Average & 19102 & 14579 & 76.3 & 11308 & 59.2 & 0 & 0.0 \\
\hline & $S D$ & 14669 & 9500 & - & 10073 & - & 0 & - \\
\hline & Median & 15273 & 15273 & - & 0 & - & 0 & - \\
\hline & IQR & $(7166,32953)$ & $(5073,23738)$ & - & $(0,11308)$ & - & $(0,0)$ & - \\
\hline \multirow[t]{10}{*}{ EgM9 } & 804 & 41573 & 6214 & 14.9 & 35359 & 85.1 & 0 & 0.0 \\
\hline & 808 & 59 & 8 & 13.6 & 51 & 86.4 & 0 & 0.0 \\
\hline & 848 & 13262 & 13262 & 100.0 & 0 & 0.0 & 0 & 0.0 \\
\hline & 849 & 2933 & 2933 & 100.0 & 0 & 0.0 & 0 & 0.0 \\
\hline & Average & 14457 & 5604 & 38.8 & 8853 & 61.2 & 0 & 0.0 \\
\hline & $S D$ & 18946 & 5700 & - & 24967 & - & 0 & - \\
\hline & Median & 8098 & 4574 & - & 26 & - & 0 & - \\
\hline & IQR & $(778,34495)$ & $(739,11500)$ & - & $(0,26532)$ & - & $(0,0)$ & - \\
\hline & Redu $\%^{\mathrm{a}}$ & 47.0 & 59.3 & - & -100 & - & 0 & - \\
\hline & $P$ value ${ }^{b}$ & 0.624 & 0.142 & - & 0.788 & - & 1.000 & - \\
\hline \multirow[t]{11}{*}{ EgM123 } & 803 & 5729 & 5729 & 100.0 & 0 & 0.0 & 0 & 0.0 \\
\hline & 806 & 12058 & 12058 & 100.0 & 0 & 0.0 & 0 & 0.0 \\
\hline & 812 & 28 & 28 & 100.0 & 0 & 0.0 & 0 & 0.0 \\
\hline & 847 & 1653 & 301 & 18.2 & 1352 & 81.8 & 0 & 0.0 \\
\hline & 850 & 23 & 23 & 100.0 & 0 & 0.0 & 0 & 0.0 \\
\hline & Average & 3898 & 3628 & 93.1 & 270 & 34.7 & 0 & 0.0 \\
\hline & $S D$ & 5123 & 5303 & - & 604 & - & 0 & - \\
\hline & Median IQR & $1653(26,8894)$ & $301(26,8894)$ & - & $0(0,676)$ & - & $0(0,0)$ & - \\
\hline & Redu\% ${ }^{a}$ & 89.2 & 98.0 & - & 0 & - & 0 & - \\
\hline & IQR & $(26,8894)$ & $(26,8894)$ & & $(0,676)$ & & $(0,0)$ & \\
\hline & $P$ value $^{\mathrm{b}}$ & 0.047 & 0.047 & - & 0.368 & - & 1.000 & - \\
\hline
\end{tabular}

${ }^{a}$ Compared to those in the GST group. Redu, reduction = (worm burden median in GST-worm burden median in the experiment group)/worm burden median in GST group $\times 100 \%$. ${ }^{\text {b }}$ The Mann-Whitney $U$ test was used to compare the worm burden median. $P$ value less than 0.05 means that reduction is significant between GST and experimental groups using median analysis. The meaning of ${ }^{a}$, ${ }^{\text {is }}$ the same in the following tables

evident in worm burdens between EgM9 vaccinated dogs and the GST control vaccinated dogs $(P>0.05)$. However, in the control group $16.5 \%$ (range from $7.5 \%$ to $22.7 \%$ ) of worms developed to the mature adult stage, compared with only $0.8 \%$ (range from $0 \%$ to $1.6 \%$ ) of worms developing to the mature stage in the experimental group. Fewer worms (17.1\%) developed 4 segments in the EgM9 vaccinated dogs compared with, $41.1 \%$ of the worms with 4 segments in the control dogs $(P=0.004 ;$ Table 1$)$. The EgM9-GST vaccine induced significant protective efficacy in terms of inhibition of worm growth and suppression of egg production after 45 days post-challenge infection compared with the control dogs.

We tested another two recombinant proteins, EgM4 and EgM123, mixed with Quil A as adjuvant in a second vaccine trial using a local breed of dog from Xinjiang. EgM123-GST induced significant protective efficacy in terms of worm burden reduction and suppression of worm growth and egg production at day 45 postinfection. No worms developed to the 4 segment stage and no worms produced eggs. In contrast, $42.5 \%$ of the worms from the control dogs had 4 segments and $16.1 \%$ 
of the worms harboured eggs (Table 2). Similar to our previous study [7], dogs vaccinated with EgM4-GST did not induce any significant protective efficacy (Table 2).

Similar to when emulsified with Freund's adjuvant, the EgM9-GST and EgM123-GST recombinant proteins mixed with Quil A induced a significant level of protective efficacy in Beagle dogs against E. granulosus infection at day 45 post-challenge infection in terms of inhibiting worm growth and suppressing egg production. This supports EgM9 and EgM123 as being encouraging candidates for future vaccine development against $E$. granulosus infection in dogs. As the egg stage is the primary cause of echinococcosis in humans and animals, suppression of egg production in canine definitive hosts will reduce or prevent echinococcosis transmission.

Also, similar to Freund's adjuvant, the EgM proteins mixed with Quil A stimulated a significant IgG1 and IgG2 response (Fig. 1), indicating IgG responses induced by

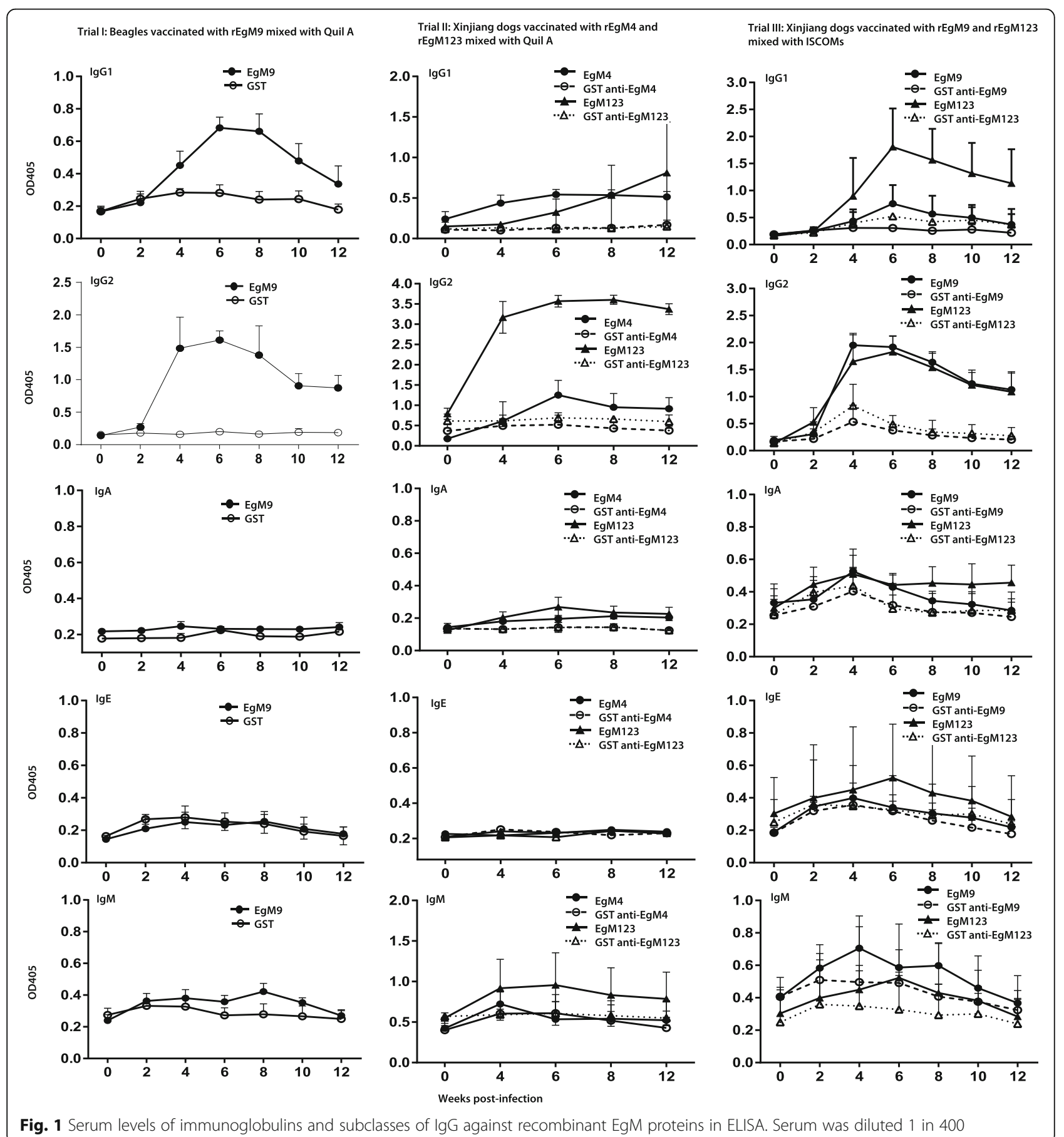


EgM are adjuvant-independent. IgG2 is likely an antibody associated with host protection against infection. However, the antibody levels dropped quickly after challenge infection to a low level at week 6 post-infection, which is the time of reproductive organ maturation and egg production. Determination of the correlation between IgG levels and worm burdens may help in an understanding of the mechanisms underpinning the elicited protective efficacy against worm infection in dogs.

\section{Vaccine efficacy of EgM proteins combined with ISCOMs}

To determine whether ISCOMs could increase the protective efficacy, we mixed them with EgM9-GST and EgM123-GST and immunized dogs of the local breed purchased from Xinjiang villages. No worms developed to the mature stage in either the experimental or control groups by day 45 post-infection. However, EgM123-GST reduced significantly the worm burden $(P<0.05)$ compared with the worm burden in the control dogs (Table 3). The worm burden in the EgM9 group were reduced by $47 \%$ (median data analysis) compared with the control group. However, statistical analysis showed no significant difference between the EgM9-GST vaccinated group and the GST vaccinated control group (Table 3). EgM9 andrEgM123 combined with the ISCOMs also induced an IgG2- predominant response (Fig. 1).

ISCOMs are a type of adjuvant that can enhance protective immunity by antigen presentation. We hypothesized that the protection induced by the EgM proteins against $E$. granulosus infection in dogs could be increased by using ISCOMs as an adjuvant. However, in the trial no worms developed to the mature stage in either the experimental or control groups after 45 days post-infection. It is possible that ISCOMs may induce protective efficacy non-specifically resulting in inhibited worm growth.

\section{Vaccine longevity study}

We also performed a vaccine longevity study to test the duration of protection induced by the EgM proteins. The numbers of E. granulosus eggs were counted and recorded using 3-5 g of dog faeces. We trialled several methods for counting fecal eggs, including a brine flotation method, but found that the method of flotation of E. granulosus eggs with saturated sucrose was repeatable and reliable (data not shown) and was used in the vaccine longevity trial.

We counted egg numbers in $3-5 \mathrm{~g}$ of faecal samples collected from day 40 post challenge infection (p.i.). E. granulosus eggs did not appear until 49 day p.i in two dogs from the PBS/Quil A group, two dogs from the EgM9/Quil A group, and one dog from the EgM123/ Quil A group (Additional file 2: Table S1). From day 51 and day 53 p.i, we did not find any eggs in any of the dog faecal 1 samples collected. However, in some dogs eggs re-appeared on day 55 p.i; seven out of ten dogs in the PBS/Quil A group released eggs ranging from 0.75 EPG to 436 EPG with an average EPG of 77, two dogs released eggs (266 EPG in one dog and 1 EPG in the other) in the EgM123/Quil A group and one dog in the EgM9/Quil A group had an EPG of 0.3 (Additional file 2: Table S1).

Over the entire time course of egg release from the dogs, there were three peaks (Fig. 2, Additional file 2: Table S1). The first occurred over the period day 55-62 p.i., the second occurred at days 65-76 p.i and the third was between day 127 and day 151 p.i. However, the egg counts were highly variable both daily and between dogs. Considering that environmental pressure is due to eggs being released from dogs, we summed all egg counts from each dog as one sample and compared the EPG counts in the two experimental groups with those in the control group, then analyse the differences by Mann-Whitney $U$ test. The analysis showed lower EGP

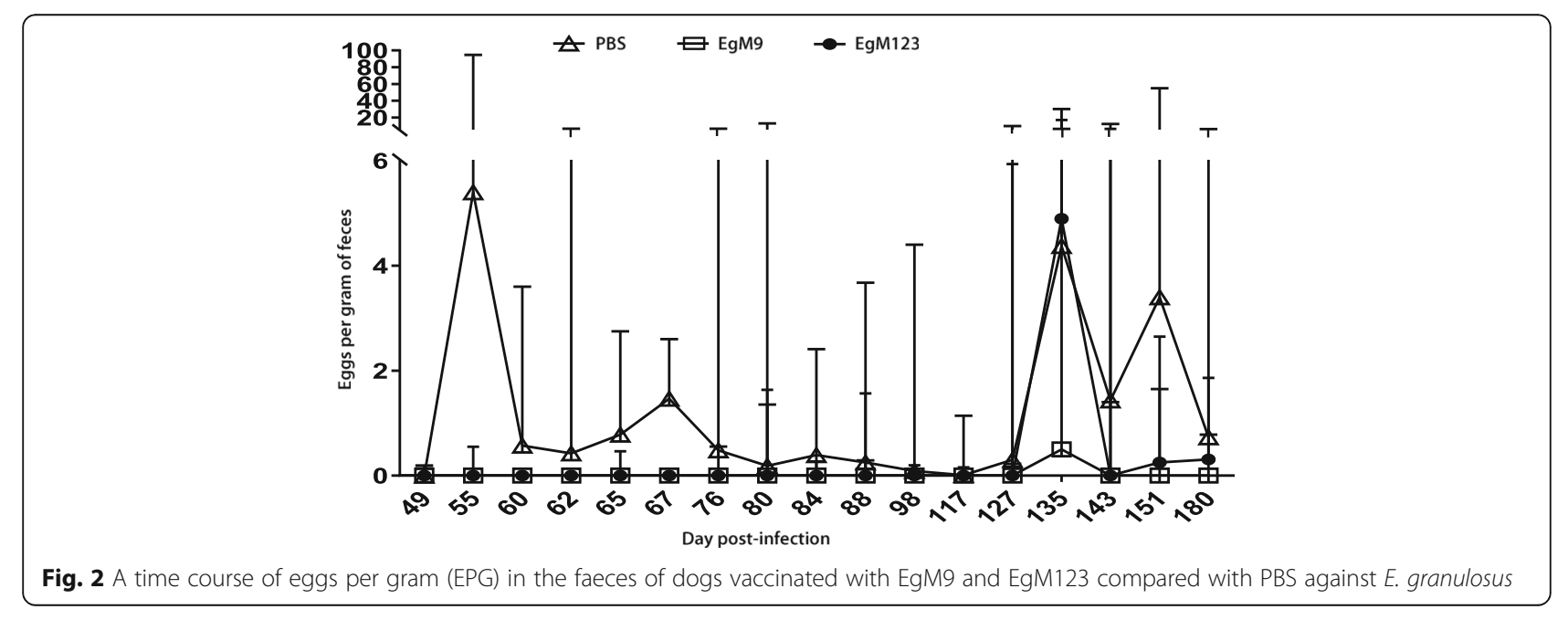


counts in EgM9/Quil A group $(P<0.05)$, whereas EgM123/Quil A group had no difference $(P>0.05)$ compared with the counts in control group. However, we found that EgM123/Quil A group released less eggs before 127 days p.i, $(P<0.05)$, indicating that this protein has partial protection efficacy in term of egg suppression.

\section{Conclusions}

EgM123 and EgM9 mixed with Quil A adjuvant resulted in a similar level of immunogenicity to these proteins emulsified with Freund's adjuvants, and induced similar levels of protective efficacy in dogs against $E$. granulosus challenge infection. These outcomes reinforce the suitability of EgM123 and EgM9 as vaccine candidates against $E$. granulosus in dogs.

\section{Additional files}

Additional file 1: Multilingual abstract in the five official working languages of the United Nations. (PDF $522 \mathrm{~kb}$ )

Additional file 2: Table S1. A time course of eggs pre gram (EPG) in the faeces of dogs vaccinated with EgM9 and EgM123 compared with PBS against E. granulosus. (XLS $234 \mathrm{~kb}$ )

\section{Abbreviations}

IQR: Interquartile range; ISCOMs: Immunostimulating complexes

\section{Acknowledgements}

We thank Dr. Yan-Ling Zheng and Dr. Jiang-Hong Dai for help in statistical analysis, and Ms. Qin-Feng Jia and Mr. Shu-Tong Li for assistance of animal management.

\section{Funding}

The project was financially supported by NSFC (China) projects (U1303203) and National Key R\&D Programs of China (2017YFD0501301).

\section{Availability of data and materials}

All the data supporting our findings have been presented in this paper.

\section{Authors' contributions}

ZZZ, GG and JL designed the experimental program and wrote the first draft; BXS, LZ and GG collected the data and finalised the statistical analysis; BPG, XZ, JWW, XTZ, WJQ and LH participated in the experiment process, including animal anatomy, immune experiments, cell count and so on. WBZ took part in the analyses, interpretation, and critically revised the paper. All authors read and approved the final paper.

\section{Ethics approval}

All protocols for using dogs were approved by the Ethical Committee of Xinjiang Academy of Animal Sciences (approval xjxmkxyAEC20060307), and the Ethical Committee of The First Affiliated Hospital of Xinjiang Medical University (approval IACUC-2015).

\section{Competing interests}

The authors declare that they have no competing interests.

\section{Author details}

${ }^{1}$ Xinjiang Veterinary Research Institute, Xinjiang Academy of Animal Sciences, 726 Dongrong Street, North Gaoxin District, Urumqi 830011, Xinjiang, China. ${ }^{2}$ State Key Laboratory of Pathogenesis, Prevention and Treatment of High Incidence Diseases in Central Asia, Clinical Medicine Institute, The first Affiliated Hospital of Xinjiang Medical University, Urumqi 830054, Xinjiang, China.
Received: 19 September 2017 Accepted: 16 April 2018

Published online: 13 June 2018

\section{References}

1. McManus DP, Gray DJ, Zhang W, Yang Y. Diagnosis, treatment, and management of echinococcosis. BMJ. 2012;344:e3866.

2. $\quad$ McManus DP, Zhang W, Li J, Bartley PB. Echinococcosis. Lancet. 2003;362: 1295-304.

3. WHO (2012) Accelerating work to overcome the global impact of neglected tropical diseases- a roadmap for implementation. http://www.who.int/ neglected_diseases/NTD_RoadMap_2012_Fullversion.pdf.

4. Craig PS, Larrieu E. Control of cystic echinococcosis/hydatidosis: 1863-2002. Adv Parasitol. 2006;61:443-508.

5. Zhang W, McManus DP. Vaccination of dogs against Echinococcus granulosus: a means to control hydatid disease? Trends Parasitol. 2008;24: 419-24.

6. Zhang W, Li J, You H, Zhang Z, Turson G, et al. A gene family from Echinococcus granulosus differentially expressed in mature adult worms. Mol Biochem Parasitol. 2003;126:25-33.

7. Zhang W, Zhang Z, Shi B, Li J, You H, et al. Vaccination of dogs against Echinococcus granulosus, the cause of cystic hydatid disease in humans. I Infect Dis. 2006;194:966-74.

8. Zhang W, Zhang Z, Yimit T, Shi B, Aili H, et al. A pilot study for control of hyperendemic cystic hydatid disease in China. PLoS Negl Trop Dis. 2009;3:e534

\section{Ready to submit your research? Choose BMC and benefit from:}

- fast, convenient online submission

- thorough peer review by experienced researchers in your field

- rapid publication on acceptance

- support for research data, including large and complex data types

- gold Open Access which fosters wider collaboration and increased citations

- maximum visibility for your research: over $100 \mathrm{M}$ website views per year

At BMC, research is always in progress.

Learn more biomedcentral.com/submissions 